\title{
Key role of piRNAs in telomeric chromatin maintenance and telomere nuclear positioning in Drosophila germline
}

Elizaveta Radion', Valeriya Morgunova', Sergei Ryazansky', Natalia Akulenko', Sergey Lavrov', Yuri Abramov', Pavel A. Komarov ${ }^{1,2}$, Sergey I. Glukhov' ${ }^{1}$ Ivan Olovnikov ${ }^{1}$ and Alla Kalmykova ${ }^{1 *}$ (D)

\begin{abstract}
Background: Telomeric small RNAs related to PIWI-interacting RNAs (piRNAs) have been described in various eukaryotes; however, their role in germline-specific telomere function remains poorly understood. Using a Drosophila model, we performed an in-depth study of the biogenesis of telomeric piRNAs and their function in telomere homeostasis in the germline.

Results: To fully characterize telomeric piRNA clusters, we integrated the data obtained from analysis of endogenous telomeric repeats, as well as transgenes inserted into different telomeric and subtelomeric regions. The small RNAseq data from strains carrying telomeric transgenes demonstrated that all transgenes belong to a class of dual-strand piRNA clusters; however, their capacity to produce piRNAs varies significantly. Rhino, a paralog of heterochromatic protein 1 (HP1) expressed exclusively in the germline, is associated with all telomeric transgenes, but its enrichment correlates with the abundance of transgenic piRNAs. It is likely that this heterogeneity is determined by the sequence peculiarities of telomeric retrotransposons. In contrast to the heterochromatic non-telomeric germline piRNA clusters, piRNA loss leads to a dramatic decrease in HP1, Rhino, and trimethylated histone H3 lysine 9 in telomeric regions. Therefore, the presence of piRNAs is required for the maintenance of telomere chromatin in the germline. Moreover, piRNA loss causes telomere translocation from the nuclear periphery toward the nuclear interior but does not affect telomere end capping. Analysis of the telomere-associated sequences (TASs) chromatin revealed strong tissue specificity. In the germline, TASs are enriched with HP1 and Rhino, in contrast to somatic tissues, where they are repressed by Polycomb group proteins.

Conclusions: piRNAs play an essential role in the assembly of telomeric chromatin, as well as in nuclear telomere positioning in the germline. Telomeric arrays and TASs belong to a unique type of Rhino-dependent piRNA clusters with transcripts that serve simultaneously as piRNA precursors and as their only targets. Telomeric chromatin is highly sensitive to piRNA loss, implying the existence of a novel developmental checkpoint that depends on telomere integrity in the germline.
\end{abstract}

Keywords: Telomere, Germline, Retrotransposon, Transgene, piRNA cluster, HP1, Rhino, Chromatin, Transcription, Drosophila

\footnotetext{
*Correspondence: allakalm@img.ras.ru

${ }^{1}$ Institute of Molecular Genetics, Russian Academy of Sciences, Kurchatov

sq. 2, Moscow, Russia 123182

Full list of author information is available at the end of the article
} 


\section{Background}

Telomere transcription is an evolutionarily conserved feature of eukaryotic telomeres [1]. The biogenesis of telomeric transcripts has been shown to be tightly linked to telomere length control and formation of telomeric chromatin. Telomeric transcripts serve as precursors of small RNAs (tel-sRNAs) discovered in mammalian embryonic stem cells, in the ciliate Tetrahymena thermophila, in plants, and in Diptera [2-5]. Small RNAs generated by the subtelomeric regions in fission yeast, as well as certain tel-sRNAs, have been implicated in the assembly of telomeric heterochromatin $[2,5,6]$. Plant and mammalian tel-sRNAs are related to the class of Piwi-interacting RNAs (piRNAs) that are generated in the germline and in stem cells $[7,8]$. However, the role of tel-sRNAs in germline-specific telomere function is poorly understood. Telomeric piRNAs and their role in telomere length control were first described in Drosophila melanogaster [3]. Using a Drosophila model, we performed an in-depth study of the biogenesis and function of the telomeric piRNAs in the germline.

The piRNA-mediated pathway provides silencing of transposable elements (TE) in the germline [7, 9]. In contrast to small interfering RNAs (siRNA), which are processed by the Dicer endonuclease from double-strand RNAs, piRNAs are generated from long single-stranded precursors. These piRNA precursors are encoded by distinct genomic regions that are enriched in damaged TE copies and termed piRNA clusters [10]. The dualstrand piRNA clusters found in the Drosophila germline produce piRNAs from precursors transcribed by both genomic strands. Distinct chromatin components of the piRNA clusters that couple transcription and RNA transport appear to direct the cluster-derived transcripts into the piRNA processing machinery [11-13]. The germlinespecific homolog of HP1-Rhino (Rhi) -is essential for piRNA production from the dual-strand piRNA clusters [14-16]. piRNAs are required at early embryonic stages for deposition of Rhi and histone 3 lysine 9 trimethylation marks (H3K9me3) at the dual-strand piRNA clusters, but at later developmental stages, the chromatin of piRNA clusters is maintained by an unknown Piwi-independent mechanism [17].

Dual-strand piRNA clusters can be classified into several types according to their structure and location: extended pericentromeric TE-enriched regions [10], individual euchromatic TE copies [18], and transgenes which contain complementarity to endogenous piRNAs $[19,20]$. In all cases, the clusters produce piRNAs that target complementary sequences located elsewhere in the genome.

The telomeres of $D$. melanogaster are maintained by transpositions of specialized telomeric retrotransposons, while the telomerase gene has likely been lost in an ancestor of Diptera [21]. The non-LTR HeT-A, TART, and TAHRE retroelements are organized in tandem head-totail telomeric arrays, with $H e T-A$ being the most abundant [22-24]. Telomere-associated sequences (TASs) consist of complex satellite-like repeats and are located proximally to retrotransposon arrays. Analysis of the ovarian small RNA-seq data revealed abundant piRNAs corresponding to both genomic strands of telomeric retrotransposons and TAS [10]; thus, telomeric piRNA clusters can be formally related to the dual-strand type. However, the main feature of the telomeric piRNA clusters is that their transcripts serve both as a source of piRNAs and as their only targets simultaneously. Telomeric transcripts are processed into piRNAs that regulate telomeric TE expression and transposition rate onto chromosome ends in the germline [3,25]. Violation of the balance between the levels of piRNAs and mRNAs encoded by telomeric retrotransposons can lead to disruption of telomere length control $[3,26]$. It is clear that involvement in piRNA production should considerably affect telomere biology in the germline; however, telomeres have not been characterized as piRNA clusters to date. In previous studies, telomeric retrotransposons were generally included in the canonical set of TEs used to evaluate the impact of piRNA pathway mutation on TE expression and piRNA production. These data demonstrate that HeT-A and related TAHRE elements are extremely sensitive to piRNA pathway disruption showing up to 1000 -fold overexpression in contrast to TART , which demonstrates only modest upregulation $[3,25$, 27, 28]. Therefore, measurement of $H e T-A$ expression has often been used as a readout of piRNA pathway disruption. However, $H e T-A$ elements are not typical piRNA targets, since they are also the source of piRNAs. Several studies have indicated differences between transcriptional regulation of telomeric and non-telomeric piRNA clusters. Indeed, in contrast to $H e T-A$, the level of the piRNA precursors transcribed by non-telomeric piRNA clusters decreases upon piRNA pathway disruption [14, $15,29]$.

Unique mapping of small RNA reads is the major source of information about the genomic origin of piRNAs; however, in the case of telomeres, it is technically challenging. Artificial sequences inserted into the endogenous piRNA clusters serve as unique marks that enable exploration of the highly repetitive genomic loci. Transgenic Drosophila strains carrying P-element constructs in the terminal retrotransposon array have been identified and characterized [30]. In contrast to the TASs that demonstrate Polycomb group (PcG) protein-mediated silencing of transgenes inserted in these regions [31-33], the telomeric retrotransposon arrays show euchromatic 
characteristics. Transgene reporters inserted in $H e T-A-$ TART-TAHRE arrays are active in somatic tissues, which have allowed for selection of such transgenic strains [30]. Therefore, based on differing abilities to silence the integrated transgenes, two telomeric subdomains were defined within Drosophila telomere in somatic tissues, namely, transcriptionally active retrotransposon arrays and heterochromatic TAS [30, 31].

Taking advantage of the telomeric transgene insertions in combination with experiments on endogenous telomeric elements, we investigated piRNA production and chromatin structure of different telomeric loci in the ovaries of transgenic flies. It was shown that telomerespecific piRNAs significantly affect the chromatin structure and expression of different telomeric regions in the germline. In contrast to somatic tissues, the TAS and HeT-A-TART-TAHRE arrays show similar chromatin structure and transcriptional status in the germline and can be related to the piRNA-producing domain. At the same time, we found that piRNA production is not similar between the transgenes integrated in different telomeric retrotransposons. Chromatin and cytological studies provide convincing evidence that telomeric piRNA clusters are highly sensitive to piRNA loss in contrast to the heterochromatic non-telomeric dual-strand piRNA clusters. Moreover, piRNA loss causes telomere translocation from the nuclear periphery toward the nuclear interior. Our data, in combination with the previously observed differences in behavior of telomeric and non-telomeric piRNA clusters $[17,29,34]$, suggest that a distinct type of piRNA cluster protects telomere integrity in the Drosophila germline.

\section{Results}

Transgenes located at different positions in telomeres produce small RNAs in Drosophila ovaries

It is well known that transgenes inserted within TASs produce abundant piRNAs and exert piRNA-mediated silencing of the complementary targets [35-38]. However, the piRNA production capacity of transgenes located within telomeric retrotransposon arrays has not been investigated to date. In this study, we used four available transgenic EY strains on a $y^{1} w^{67 c 23}(y w)$ strain background carrying the $\mathrm{P}\{\mathrm{EPgy} 2\}$ construct in the telomeric retrotransposon arrays [30]. $\mathrm{P}\{\mathrm{EPgy} 2\}$ is a $P$-element-based vector containing mini-white and yellow genes. The transgene EY08176 was inserted into the GAG ORF of $H e T$-A-related TAHRE on chromosome 2R. The transgenes EY00453 and EY00802 were integrated into 3' UTR of TART-B1 on 3L, while the EY09966-into TART-C on the fourth chromosome. All TART insertions were located in the $3^{\prime} \mathrm{UTR}$ within the long non-terminal repeats containing the promoter region. Insertion sites are located between the sense and antisense transcription start sites [39], which seems to be a hot spot for insertions [30]. All transgenes were mapped at 12-23 kb from TASs [30]. The orange eye color of the EY08176, EY00453, and EY00802 transgenic flies corresponds to the previously reported phenotype and indicates a high level of the mini-white reporter gene expression [30, 33]. The EY03383 strain carries P\{EPgy 2$\}$ in $2 \mathrm{R}$ TAS [30]. The insertions in the TAS (EY03383) and in the telomere of the fourth chromosome (EY09966) are silenced and demonstrate a white or variegated eye color phenotype (Table 1). The euchromatic EY03241 transgene was used as a non-telomeric control. Insertion locations are shown schematically in Fig. 1a, b. DNA FISH on polytene chromosomes of salivary glands confirmed the telomeric localization of transgenes (Additional file 1: Figure S1). Short names of telomeric insertions are indicated in Fig. 1c.

Abundant endogenous HeT-A, TAHRE, and TART -specific small RNAs are found in $y w$ and transgenic strains (Fig. 1a; Additional file 1: Figure S2); however, it is unclear how each particular telomeric element copy contributes to the production of piRNAs. To address

Table 1 Comparison of telomeric transgene properties in somatic tissues and in the germline

\begin{tabular}{|c|c|c|c|c|c|c|c|c|c|}
\hline \multirow[t]{2}{*}{ Strain [30] } & \multirow[t]{2}{*}{ Insertion site } & \multicolumn{2}{|c|}{ Somatic tissues [30, 31, 33] } & \multicolumn{6}{|c|}{ Female germline } \\
\hline & & Eye color & Chromatin state & $\begin{array}{l}\text { piRNA } \\
\text { production }\end{array}$ & Rhi & HP1a & H3K9me3 & $\begin{array}{l}\text { Mini-white } \\
\text { transcription }\end{array}$ & Chromatin state \\
\hline EY08176 & $\begin{array}{l}\text { TAHRE Gag, chr.2R } \\
\text { telomere }\end{array}$ & Orange & $\begin{array}{l}\text { Active telomeric } \\
\text { domain }\end{array}$ & +++ & +++ & +++ & +++ & + & $\begin{array}{l}\text { Dual-strand piRNA } \\
\text { cluster }\end{array}$ \\
\hline EY00802 & TART-B, $3^{\prime}$ regula- & & & + & + & +++ & +++ & + & \\
\hline EY00453 & $\begin{array}{l}\text { tory region, chr.3R } \\
\text { telomere }\end{array}$ & & & + & + & +++ & +++ & + & \\
\hline EY09966 & $\begin{array}{l}\text { TART-C, } 3^{\prime} \text { regula- } \\
\text { tory region, chr.4 } \\
\text { telomere }\end{array}$ & White & $\begin{array}{l}\text { Repressed telomeric } \\
\text { domains }\end{array}$ & + & + & +++ & +++ & + & \\
\hline EY03383 & TAS, chr.2R & Variegation & & +++ & ++ & +++ & +++ & + & \\
\hline
\end{tabular}


this question, we sequenced small RNAs from ovaries of five telomeric transgenic strains and the EY03241 strain (control) with a euchromatic insertion. The euchromatic transgene EY03241 (control) produces a negligible amount of small RNAs (Fig. 1c, Additional file 2: Table S1). In contrast, all telomeric transgenes produce small RNAs; however, the levels vary considerably between transgenes (Fig. 1c), which may be attributed to variation in piRNA production between the integration sites. The small RNAs are mapped to both genomic strands of the entire transgene EY08176 (insTAHRE). Moreover, most of these small RNAs are 24-29 nt long and demonstrate $5^{\prime}$ terminal uridine bias (1U bias), which is a characteristic of the piRNAs (Fig. 1d). We found the sense/antisense piRNA pairs (relative to transgene) overlapping by $10 \mathrm{nt}$, which is a signature of the ping-pong piRNA amplification cycle $[10,40]$ (Fig. 1e). Such characteristics of transgenic small RNAs strongly suggest that the transgene is integrated within the pre-existing piRNA cluster. The EY03383 transgene inserted into the dual-strand piRNA cluster within the $2 \mathrm{R}$ TAS also produces abundant piRNAs from both genomic strands (Fig. 1c), similar to the transgenes integrated into subtelomeric piRNA clusters on the $\mathrm{X}$ and $3 \mathrm{R}$ chromosomes $[19,34$, 38].

The EY00453, EY00802, and EY09966 (insTART ) transgenes produce fewer small RNAs compared to EY08176 (insTAHRE) and EY03383 (insTAS) but apparently more than the EY03241 (control) transgene (Fig. 1c, Additional file 2: Table S1). A significant fraction of small RNAs produced by TART insertions are 21-nt siRNAs; no ping-pong signal was detected for the transgenic piRNAs showing $1 \mathrm{U}$-bias. Interestingly, the production of 21-nt RNAs is less variable between the telomeric transgenes than that of piRNAs (Additional file 2: Table S1). Unique mapping of the small RNAs to all telomeric transgenes revealed single-mapped piRNAs derived from the P-element fragments and linkers, confirming that the observed effects are transgene-specific (Additional file 1: Figure S3).

Northern blotting of the white-specific small RNAs from the ovaries of transgenic strains confirmed the presence of abundant small RNAs in EY08176 (insTAHRE) and EY03383 (insTAS) strains (Fig. 1f, Additional file 1: Figure S4).

Thus, all telomeric transgenes can be considered as piRNA clusters; however, the capacity to produce piRNAs varies significantly between the transgenes integrated into different telomeric sites, with TART promoter regions being considerably less productive (Table 1 ).

\section{HP1, Rhino, and H3K9me3 associate with different telomeric transgenes}

The piRNA-guided transcriptional silencing is mediated by the deposition of HP1 and H3K9me3 [29, 41-43], whereas the germline-specific HP1 homolog Rhi serves as a chromatin marker of the dual-strand piRNA clusters $[13-16,44]$. To answer the question if these chromatin components are associated with telomeric retrotransposons and transgenes in ovaries, we performed chromatin immunoprecipitation (ChIP) assay. The transcriptionally active rp49 and metRS- $m$ genes and the intergenic $60 D$ region were included in the analysis as negative controls. ChIP-qPCR using ORF-specific primers shows that endogenous HeT-A, TAHRE, and TART are enriched by HP1, Rhi, and H3K9me3 (Fig. 2). However, these data show the mean values for all telomeric copies and therefore do not reflect their possible heterogeneity. At the same time, transgene-specific primers enable us to examine the chromatin state at particular telomeric loci. Indeed, all studied transgenes were also considerably enriched in HP1 and H3K9me3, but we observed strong differences in Rhi binding among telomeric transgenes (Fig. 2). While Rhi occupancy was very high in two regions (5' P-element arm and mini-white) of EY08176 (insTAHRE), Rhi binding to TART transgenes was much

\footnotetext{
(See figure on next page.)

Fig. 1 Generation of small RNAs by telomeric transgenes. a Schematic structure of telomeric elements is shown above. Insertion sites of transgenes are indicated as triangles situated above and below the schemes, which correspond to their genomic orientation. The profiles of small RNAs in ovaries of $y w$ strain are shown along the canonical sequences of HeT-A, TAHRE, TART-A, TART-B, and TART-C telomeric retrotransposons. Normalized numbers of small RNAs (RPM, reads per million, 0-3 mismatches) in a 30-bp window were calculated. Length distribution of the telomeric element small RNAs is shown below. Percentages of reads having $1 \mathrm{U}$ are indicated for each strand (only 24-29-nt reads were considered). $\mathbf{b}$ Scheme of transgenic insertion sites in euchromatin and TAS of chromosome 2R. c Normalized numbers of small RNAs mapped to transgenic constructs (blue-sense; brown-antisense; no mismatches allowed). Mapping of piRNAs (24-29 nt) and siRNAs (21 nt) onto the transgenes is shown separately. Scheme of the P\{EPgy2\} transgene is shown above. Short names of telomeric insertions are indicated. $\mathbf{d}$ Length distribution of transgenic small RNAs. Percentage of reads having $1 \mathrm{U}$ are indicated for each strand (only 24-29-nt reads were considered). e Relative frequencies (Z-score) of 5' overlap for sense and antisense 24-29-nt piRNAs (ping-pong signature). $\mathbf{f}$ Northern blot hybridization of the RNA isolated from the ovaries of EY08176, EY03383, EY00453, EY00802, EY09966, and EY03241 strains was done with the white riboprobe to detect antisense piRNAs. Lower panel represents hybridization to mir-13b1 microRNA. P32-labeled RNA oligonucleotides were used as size markers
} 
a

a

HeT-A

TAHRE

TART-A

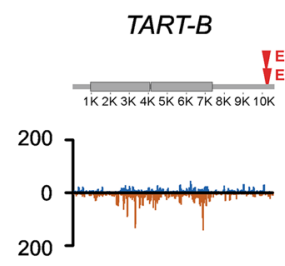

00
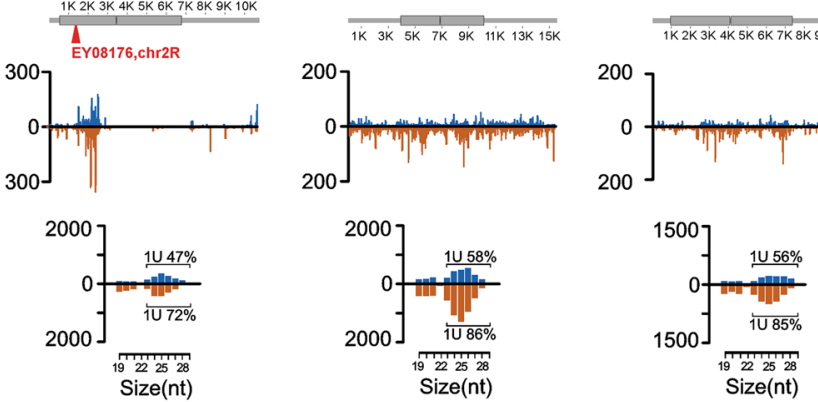

TART-C
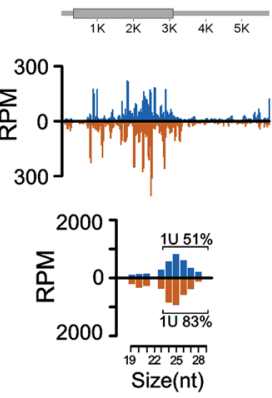

${ }^{19} 222528$

b

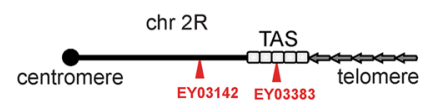

C
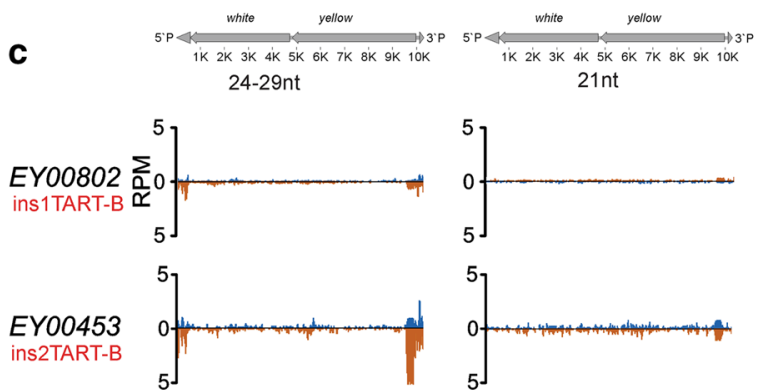

EYO9966
insTART-C
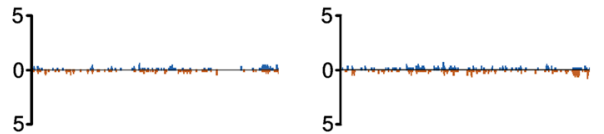

EY08176
insTAHRE
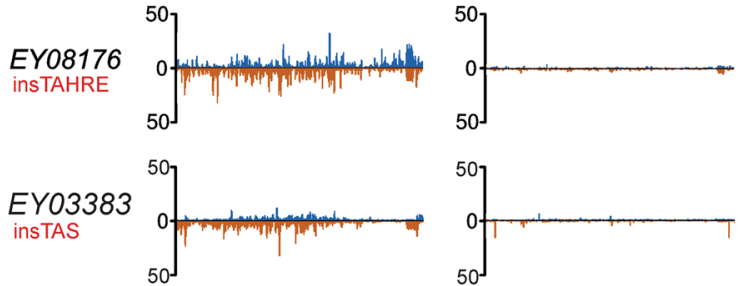

$\underset{\text { control }}{\text { EY0324 }}$
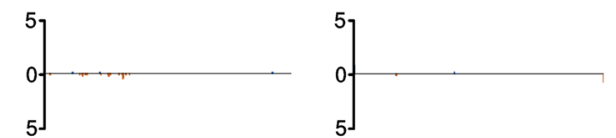

f

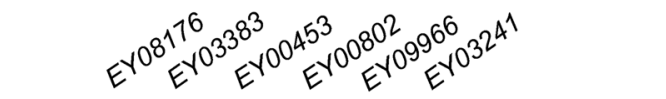

$30-$

19-

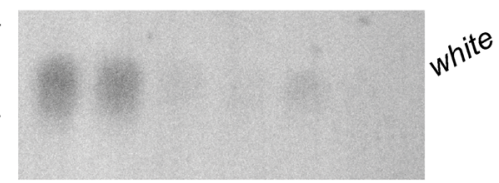

d

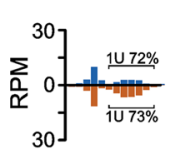

$\left.{ }^{30}\right]$

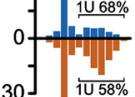

307

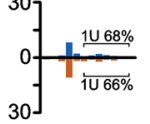

100
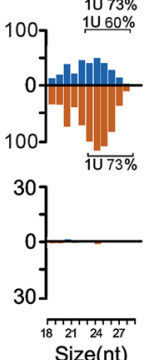

Size(nt) e
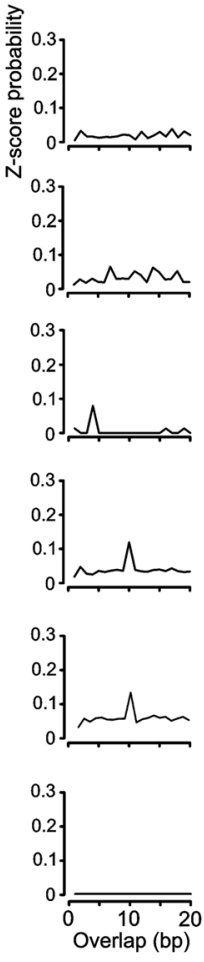
less pronounced but statistically significant (Fig. 2; Additional file 1: Figure S5).

We conclude that telomeric retrotransposon arrays are heterogeneous in piRNA production, which correlates with Rhi binding. At the same time, all telomeric transgenes, regardless of the piRNA production rate and Rhi binding, associate with HP1 and H3K9me3 in ovaries. These observations raise the question regarding the role of the piRNA pathway in deposition of HP1 and H3K9me3, which are crucial for telomere function.

\section{piRNAs are required for deposition and maintenance of HP1, Rhi, and H3K9me3 chromatin components at telomeric retrotransposon arrays in ovaries}

HP1 and H3K9me3 are important components of telomeric chromatin that are involved in the control of telomere length in different species including mammals [45]. HP1 and H3K9me3 are also present in the Drosophila telomeres in somatic tissues [31, 46, 47]; however, the mechanisms underlying their deposition at the telomere are not clear and likely differ between somatic and germline tissues. To study the role of the piRNA pathway in the deposition of HP1, H3K9me3, and Rhi at telomeres, we examined the association of these proteins with telomeric transgenes and endogenous telomeric repeats upon piRNA loss caused by depletion of the RNA helicase Spindle-E (SpnE) [3, 48]. In addition, we compared the chromatin dynamics of telomeric and non-telomeric piRNA clusters. We demonstrated that spnE mutation caused a considerable decrease in the association of HP1, Rhi, and H3K9me3 with the EY08176 telomeric transgene as well as with endogenous $H e T-A$, TAHRE, and TART-A elements accompanied by activation of their expression (Fig. 3a; Additional file 1: Figure S6). This result agrees with the previously observed loss of H3K9me3 and HP1 from $H e T-A$ upon piRNA pathway disruption $[28,29]$. In contrast to the telomeric regions, Rhi, HP1, and H3K9me3 are not displaced from $42 A B$ locus, $38 \mathrm{C1}$, and other dual-strand piRNA clusters in ovaries of spnE mutants (Fig. 3a). It is worth noting that different piRNA clusters use various mechanisms of transcriptional regulation. Transcription of most dual-strand piRNA clusters is initiated at random sites and regulated by non-canonical mechanisms. Several piRNA clusters, including $38 \mathrm{C} 1$, use flanking promoters along with internal initiation sites for piRNA precursor transcription
[15]. However, chromatin of the $38 \mathrm{C} 1$ cluster, as well as other non-telomeric clusters, is not changed in $s p n E$ mutant ovaries (Fig. 3a).

To examine the chromatin dynamics of the EY00453 transgene (ins2TART-B), we genetically combined it with the $\operatorname{spnE}$ short hairpin construct because the $s p n E$ locus is located on the same chromosome as the transgene insertion site, making it difficult to combine the transgene and $s p n E$ mutations. It is remarkable that the chromatin of this transgene is resistant to piRNA loss caused by spnE depletion (Fig. 3b), suggesting that the mechanism of chromatin maintenance at the regulatory region of TART is different from the other telomeric regions. We suggested that the TART promoter is resistant to piRNA-mediated chromatin assembly. We therefore performed ChIP-qPCR using primers specific to the endogenous TART-B promoter region in the vicinity of the insTART-B transgene insertion site. These data show that chromatin in this region is also resistant to piRNA loss caused by $s p n E$ depletion (Fig. 3b). Surprisingly, the promoter regions of TART-A elements lose HP1, Rhi, and H3K9me3 in spnE mutants (Fig. 3a). The possible explanation for the differences between ChIP data on promoter and ORF regions of endogenous TARTs related to different subfamilies is that TART copies are heterogeneous in chromatin structure and Rhi binding; however, the nature of this heterogeneity is still unclear and requires further investigation. Of note, the levels of Rhi vary at two different positions of $42 A B$, which likely reflects the intrinsic heterogeneity of the chromatin structure of natural piRNA clusters.

Thus, ChIP data suggest that the piRNA pathway provides a germline-specific mechanism for HP1, Rhi, and H3K9me3 deposition at different telomeric regions. Additionally, it is essential for maintenance of this chromatin state during gametogenesis in contrast to the nontelomeric dual-strand piRNA clusters.

\section{piRNAs are required for telomere localization at the nuclear periphery but are dispensable for telomere capping and clustering in the germline}

To verify the association of Rhi with endogenous telomeres in wild type ovaries and upon piRNA loss, we visualized HeT-A and TART using DNA FISH combined with Rhi immunostaining. In contrast to the giant polytene chromosomes of salivary glands, the chromatids

\footnotetext{
(See figure on next page.)

Fig. 2 Chromatin components of the telomeric regions. HP1, H3K9me3, and Rhi occupancies at P\{EPgy2\} transgenes were estimated by ChIP-qPCR using primers corresponding to 5'-P-element transgenic sequence. Primers corresponding to ORFs were used for the analysis of endogenous HeT-A, TART-A, and TAHRE. Two regions of the $42 A B$ piRNA cluster are enriched in all studied chromatin components. rp49, metRS- $m$, and $60 D$ regions are used as negative controls. Asterisks indicate statistically significant differences in Rhi enrichment relative to EY03241 (control) $\left({ }^{*} P<0.05\right.$ to 0.01 , ${ }^{* *} P<0.01$ to $0.001,{ }^{* *} P<0.001$, unpaired $t$ test). The difference in the HP1 binding between transgenes is statistically insignificant
} 

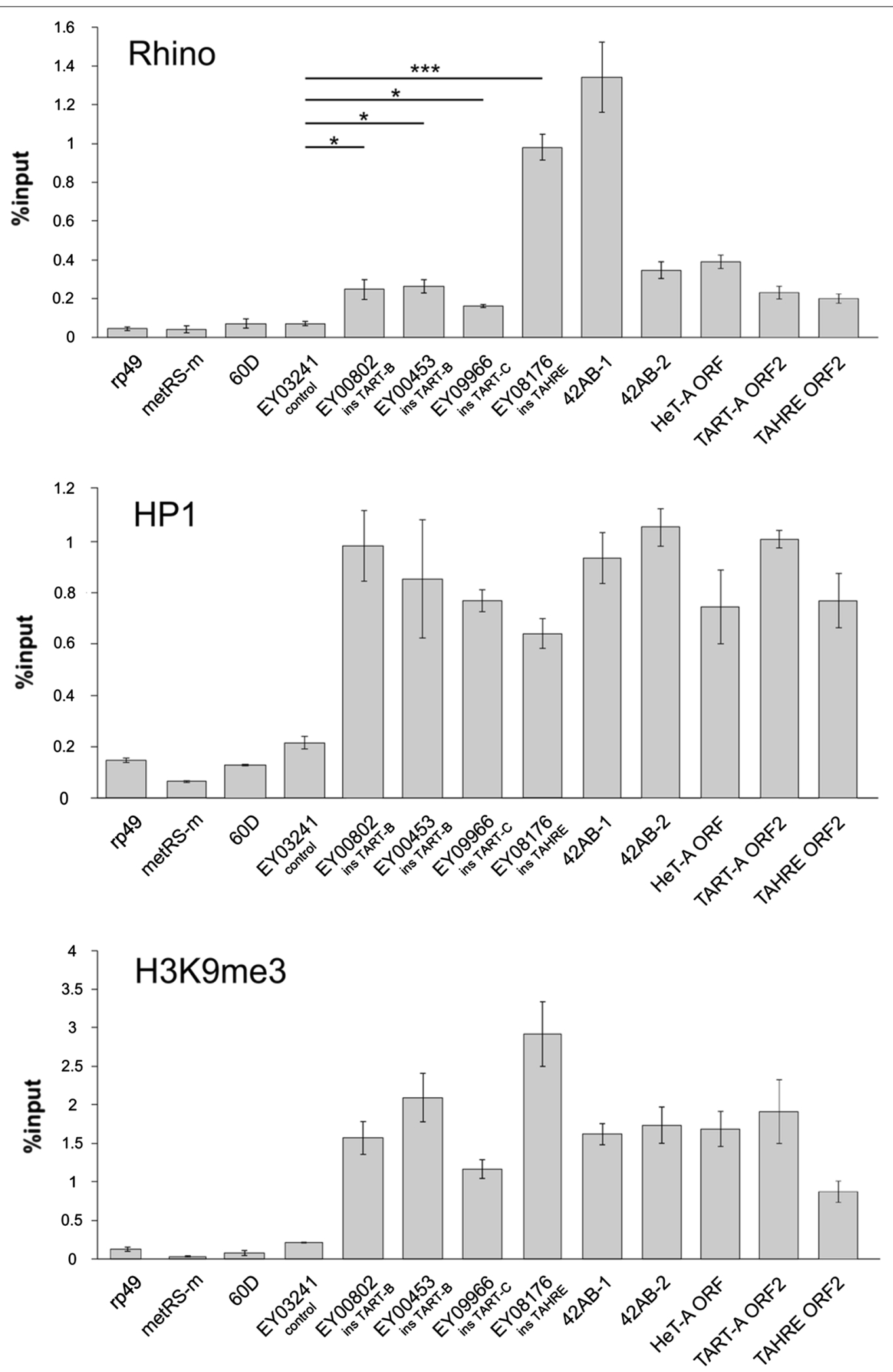

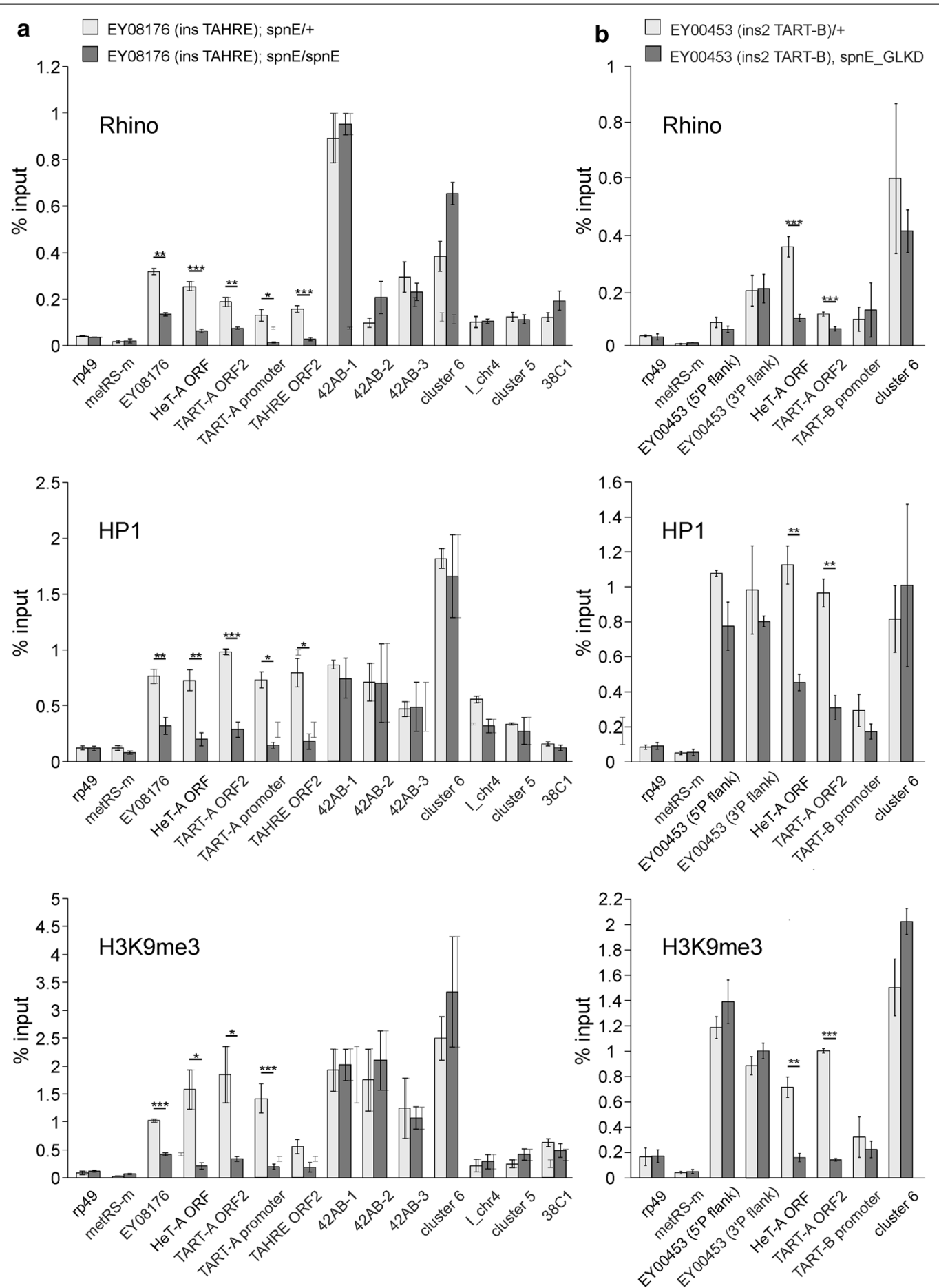

Fig. 3 Role of piRNA pathway in the deposition of HP1, Rhi, and H3K9me3 at telomeric transgenes and endogenous telomeric retrotransposons in ovaries. a ChIP-qPCR analysis of HP1, Rhi, and H3K9me3 enrichment at EY08176 transgene (insTAHRE), endogenous HeT-A, TART-A, TAHRE and a set of dual-strand piRNA clusters in ovaries of hetero- and trans-heterozygous (spn- $E^{1} /$ spn- $E^{\text {hls } 3987}$ ) spindle- $E$ mutants. Asterisks indicate statistically significant differences in chromatin protein levels at the indicated regions between spnE/+ and spnE/spnE ${ }^{*} P<0.05$ to $0.01,{ }^{*} P<0.01$ to 0.001 , ${ }^{* * *} P<0.001$, unpaired $t$ test). b ChIP-qPCR analysis of HP1, Rhi, and H3K9me3 enrichment at EY00453 (ins2TART-B), endogenous HeT-A, TART-B, and dual-strand piRNA cluster 6 in ovaries upon spnE germline knockdown. Here, we used primers specific to the transgene insertion site instead of those to $5^{\prime} P$ due to the presence of additional P-element-based constructs in the genome. The TART-B promoter was amplified using a primer pair surrounding the inSTART-B insertion 
of highly polyploid nurse cells are only partially conjugated, allowing for detection of numerous DNA FISH signals. In the ovaries of the $y w$ strain, most $H e T-A$ foci are clustered and overlap with the largest Rhi foci, forming rosette-like structures near the nuclear envelope in the different D. melanogaster strains (Fig. 4a; Additional file 1: Figure S7a). We observed that the clustered $H e T-A$ signals lose Rhi and are located toward the nuclear interior in $s p n E$ and piwi piRNA pathway gene mutants, although in zucchini (zuc) mutants these effects are less pronounced (Fig. 4a; Additional file 1: Figure S7b). According to previously published data, the same $z u c$ mutation exerted a weak effect on the abundance of $H e T-A$-specific piRNAs compared to piwi and spnE [48], even though it is the most severe allelic combination of $z u c$ [49]. Most likely, zuc function is less important in $H e T-A$ chromatin assembly than that of piwi and spnE.

Positioning of the clustered $H e T-A$ signals relative to the nuclear surface was estimated by $3 \mathrm{D}$ quantitative confocal image analysis of HeT-A DNA FISH samples on ovaries of the control, spnE, and piwi mutant flies. It was found that the distance from the center of the HeT-A FISH signal to the nuclear periphery of nurse cells increased significantly in $s p n E$ and piwi trans-heterozygous mutants compared to heterozygous controls (Fig. 4b).

Next, we addressed a question concerning the role of piRNAs in the deposition of the protective capping complex at the chromosome ends in the germline. We performed $H e T-A$ DNA FISH combined with immunostaining of HOAP - the main component of the Drosophila telomere capping complex [50] - on ovaries of control flies and spnE mutants. HOAP extensively colocalizes with the clustered and individual $H e T-A$ signals both in control and mutant nurse cell nuclei (Fig. 4c). Those HOAP signals that do not colocalize with $H e T-A$ most likely correspond to telomeres lacking full-length $H e T-A$ copies, since the HeT-A probe contains an ORF fragment. Previously, ChIP analysis showed a reduction of HeT-A enrichment by HOAP in aubergine and armitage but not in ago3 and rhi piRNA pathway gene mutants [51]. Thus, HOAP loading at telomere ends appears to be mediated by the specific piRNA pathway components but not by piRNAs.

We suggested that the differences in chromatin structure and piRNA production between the telomeric transgenes might be explained by specific features of the telomeric retroelements in which they are inserted. Using dual color DNA FISH with $H e T-A$ and TART probes corresponding to their ORFs, we showed that $H e T-A$ and TART had different distributions in the nuclei of polyploid nurse cells. Surprisingly, in contrast to the clustered $H e T-A$ foci, the majority of TART signals were separated, and only a few of them colocalized with $H e T-A$ (Fig. 4d). Most likely, this pattern can be explained by the fact that the full-length $H e T-A$ and TART are not present at all telomeres in the $y w$ strain. In addition, TART-enriched telomeres do not seem to be involved in telomere clustering, in contrast to $H e T-A$-enriched telomeres. The TART DNA FISH combined with Rhi immunostaining demonstrates that the single TART signals colocalize with small individual Rhi foci (Fig. 4e). This pattern agrees with ChIP results showing that Rhi is deposited less at endogenous TARTs and TART transgenes than at HeTAs. TAHRE DNA FISH signals overlap with large Rhi foci (Fig. 4f). Depletion of spnE led to a dramatic decrease in the colocalization of Rhi with HeT-A, TAHRE, and TART $-A$ DNA FISH signals in contrast to the $42 A B$ signals, and the latter continue to be associated with Rhi (Fig. 4; Additional file 2: Table S2).

Thus, piRNAs contribute significantly to the deposition of HP1, Rhi, and H3K9me3 at the telomeric HeT$A-T A R T-T A H R E$ arrays and to the nuclear position of telomeres in the germline. However, piRNAs play a minor role in the formation of the telomere capping complex and telomere clustering.

\section{Structure of subtelomeric chromatin in the germline} Drosophila TAS regions consist of complex satellitelike tandem repeats. In the germline, these regions produce abundant piRNAs and are related to the most potent piRNA clusters [10]. On the contrary, in somatic cells, the Drosophila TASs are enriched in H3K27me3 marks and bind Polycomb group (PcG) proteins [3133], which induces silencing of transgenic constructs

\footnotetext{
(See figure on next page.)

Fig. 4 piRNAs are required for telomere localization at nuclear periphery. a DNA FISH with HeT-A (green) combined with Rhi staining (red) was performed on ovaries of the $y w$ strain and of the $s p n-E^{1} / s p n-E^{h / 53987}$ mutants. $\mathbf{b}$ Estimation of the positioning of clustered HeT-A signals relative to the nuclear surface of nurse cells by 3D quantitative confocal image analysis of HeT-A DNA FISH on ovaries of spnE/+, spn- $E^{\top} /$ spn- $^{\mathrm{h} / \mathrm{s} 3987}$, piwi/+, and piwi /piwi $^{\mathrm{Nt}}$ mutants. $\mathbf{c}$ piRNAs are dispensable for telomere capping and telomere clustering. DNA FISH with HeT-A probe combined with HOAP staining was performed on ovaries of the $y w$ and spn- $E^{1} / s p n-E^{h / s 3987}$ strains. d Double DNA FISH with HeT-A (red) and TART (green) probes was performed on ovaries of the $y w$ strain. $\mathbf{e}, \mathbf{f}$ piRNA pathway disruption causes loss of Rhi from TART but not from the $42 A B$ piRNA cluster. DNA FISH (green) with TART-A (e) or 42AB (f) probes combined with Rhi staining (red) was performed on ovaries of the yw and spn- $E^{1} / s p n-E^{h / s 3987}$ strains. DNA is stained with DAPI (blue). Nuclei of nurse cells from VIII to X stages of oogenesis are shown
} 
a

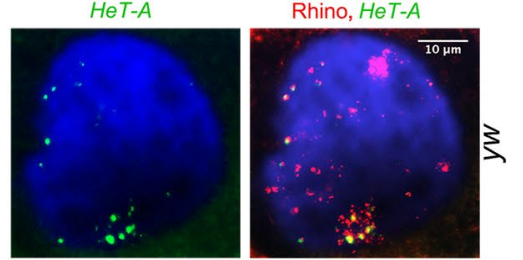

b

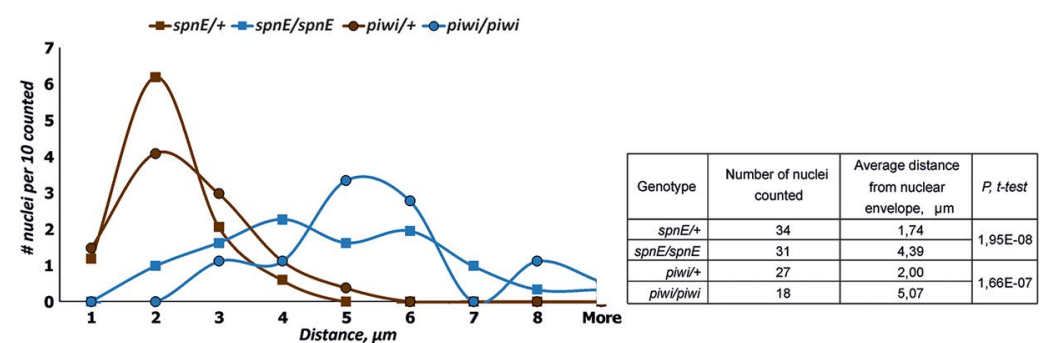

C

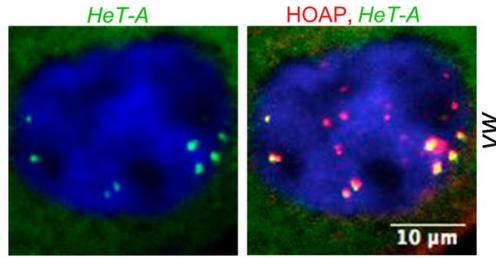

d

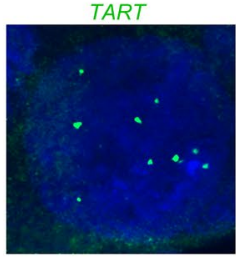

HeT-A

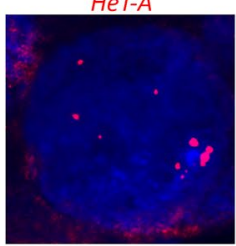

TART, HeT-A

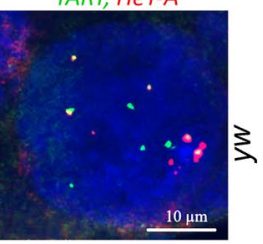

HeT-A

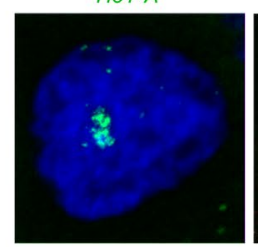

Rhino, HeT-A

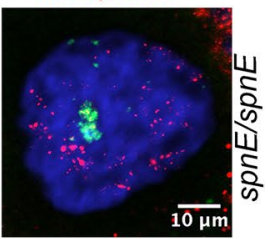

$\overline{0 \mu \mathrm{m}}$

$\frac{1}{5}$

$\frac{2}{2}$ e

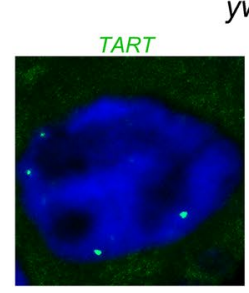

f

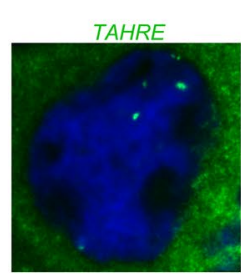

g

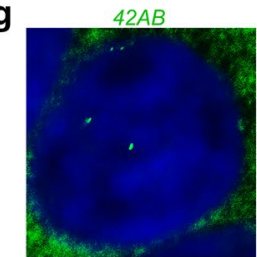


inserted in TASs, a phenomenon known as the telomeric position effect [52, 53]. Thus, the chromatin structure of TAS regions is likely to be regulated in a tissue-specific manner. Using transgene-specific primers, we performed chromatin analysis of the EY03383 transgene (insTAS) in Drosophila ovaries and observed its enrichment in HP1, H3K9me3, and Rhi (Fig. 5a) which is in accordance with the piRNA production by this transgene (Fig. 1). To examine the chromatin state of endogenous TAS in the germline, we performed TAS DNA FISH combined with immunostaining of Rhi and H3K27me3-histone modification, associated with PcG silencing. The DNA probes corresponding to the 2R-3R and 2L-3L TASs were used for FISH on the ovaries of the control $y w$ strain. TAS signals show a much stronger colocalization with Rhi than with H3K27me3 in the nuclei of nurse cells (Fig. 5b, Additional file 2: Tables S2, S3). We observed a loss of colocalization between Rhi foci and TAS signals in spnE mutants and upon piwi germline knockdown; at the same time, colocalization of H3K27me3 and TAS signals remained at a low level in the nurse cell nuclei of $s p n E$ mutants (Fig. 5b, Additional file 1: Figure S8). Thus, the PcGdependent silencing of TAS is not established in the germline, which is in contrast to somatic tissues.

Next, we compared the expression activity of telomeric transgenes in ovaries. We found that the steady-state RNA levels of mini-white reporter gene were equally low in all transgenic strains but exceeded background signal in the $y w$ strain in which white locus was partially deleted (Fig. 5c). Low levels of mini-white transcripts can be explained by a weak activity of the white promoter in the ovaries. Simultaneously, active expression of the mini-white reporter was observed in the eyes of EY08176 (insTAHRE), EY00802 (ins1TART-B), and EY00453 (ins2TART-B) transgenic strains but not in EY03383 (insTAS) and EY09966 (insTART-C) strains. Thus, the transcription activity of transgenes located at different positions within the telomere is similar in the germline but differs considerably in the somatic tissues and appears to be defined by the tissue-specific chromatin structure.

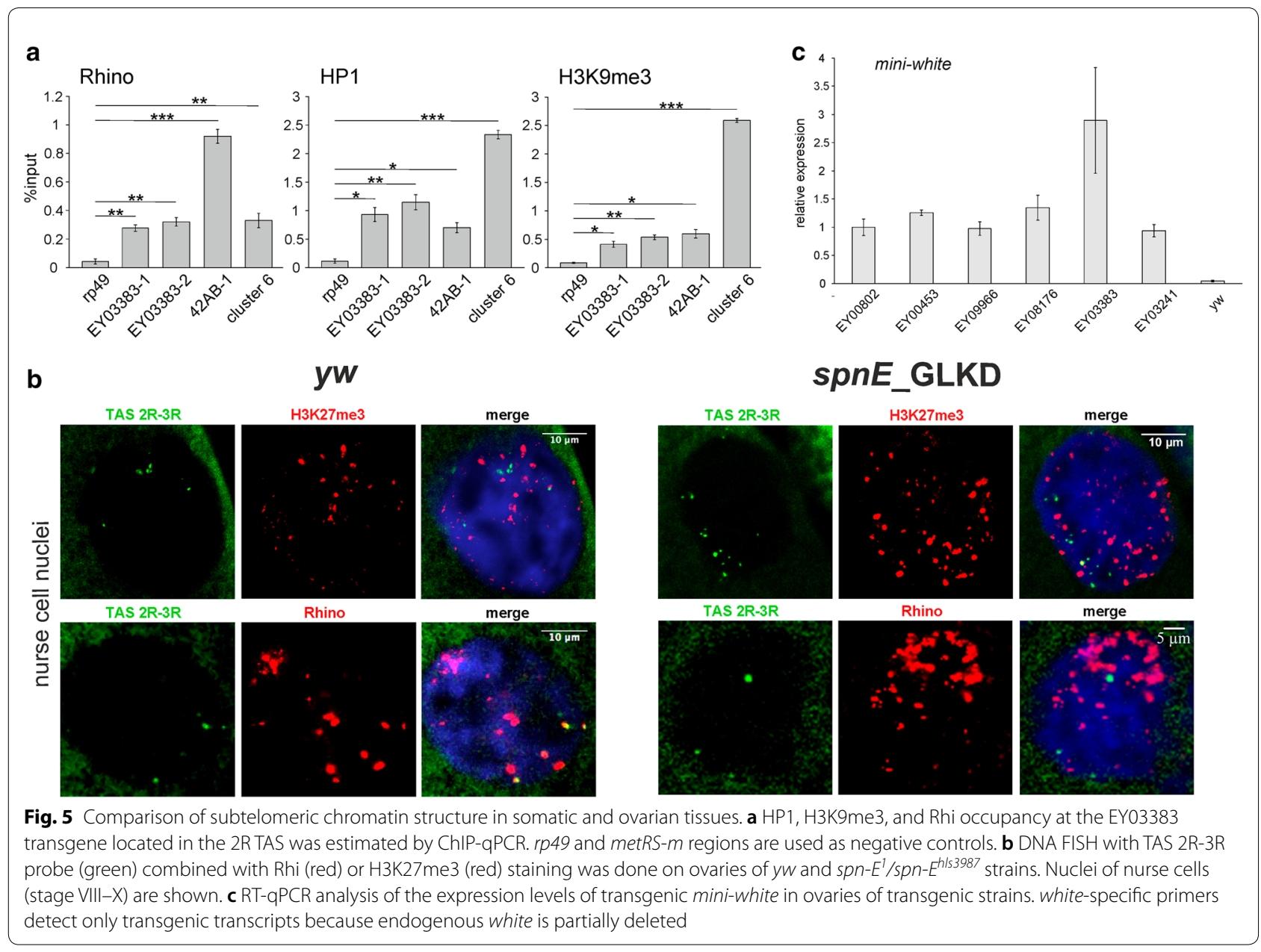




\section{Discussion}

piRNA production and Rhi binding differ along the telomeric region

In this study, we described telomeres as piRNA clusters using a combination of different approaches. The data on endogenous telomeric retrotransposons show that they produce piRNAs and associate with Rhi. Intriguingly, the piRNA production by individual telomeric transgenes depends on the type of telomeric retrotransposon in which the transgene is inserted. The transgene located within TAHRE produces considerably more piRNAs and shows stronger enrichment in Rhi than the transgenes located in the promoter region of TART elements. Accordingly, we revealed the low level of Rhi biding to the endogenous TART-B promoter region. Moreover, Rhi immunostaining and TART FISH data also demonstrate that much less Rhi is deposited on $T A R T$ in comparison with $H e T-A$, suggesting a lower susceptibility of TART elements to engagement in piRNA production in general. Most likely, TART copies are not equivalent in their capacity for piRNA production and Rhi binding, although the nature of these differences is unclear. Previously published works have already underlined the strong differences between $\mathrm{HeT}$ $A$ and TART in genomic copy number, structure, patterns of transcription, and response to piRNA pathway disruption [3, 22, 54, 55]. TART transcripts are more stable [54], which can be explained by their role in providing reverse transcriptase (RT) for the transpositions of the main structural telomeric element $H e T$ $A$ lacking RT. We suggested the intriguing possibility that the transcripts of full-length TART copies might be protected from piRNA biogenesis machinery by an unknown mechanism to ensure encoding of the crucial enzyme for telomere elongation, TART RT, in the germline.

Telomeric chromatin plays a pivotal role in telomere protection and maintenance. $\mathrm{HP} 1$ and $\mathrm{H} 3 \mathrm{~K} 9 \mathrm{me} 3 \mathrm{regu}-$ late capping, telomeric repeat silencing, and control of their transpositions onto chromosome ends [46, 47, 56]. Interestingly, all telomere insertions bind similar amounts of HP1 and H3K9me3 but strongly differ in Rhi association. Surprisingly, strong enrichment of the EY08176 transgene in Rhi, which recognizes the same H3K9me3 marks as HP1, does not abolish or significantly reduce $\mathrm{HP} 1$ binding compared to the TART insertions, indicating that Rhi and HP1 do not compete with each other for binding sites at telomeric chromatin. Thus, our data revealed heterogeneity in piRNA production and Rhi deposition within telomeric repeats. TART retrotransposons are less susceptible to Rhi binding than are $H e T-A$ and TAHRE.
Telomeric regions represent a distinct type of self-targeting dual-strand piRNA cluster

piRNA sources and piRNA targets are mainly represented by different genomic loci in the Drosophila germline. The piRNA clusters, enriched in damaged TE fragments, produce piRNAs that target active TEs $[10,29]$. The telomeric piRNA clusters have a dual nature, possessing properties of both piRNA-clusters and piRNA-targets. It is well known that the piRNA targets are silenced at the transcriptional level via the assembly of repressive chromatin; loss of piRNAs causes strong reduction of HP1 and $\mathrm{H} 3 \mathrm{~K} 9 \mathrm{me} 3$ marks at complementary targets, leading to their overexpression [25, 28, 29, 41, 42]. However, piRNA loss fails to activate non-telomeric piRNA cluster transcription and switching from a repressive to an active chromatin state $[15,17,29]$.

In-depth analysis of the telomeric piRNA clusters revealed strong differences in chromatin dynamics between telomeric and non-telomeric piRNA clusters. Using different approaches, we demonstrated that the piRNA pathway mutations induce loss of HP1, H3K9me3, and Rhi from the telomeric transgene located in the TAHRE-HeT-A arrays, as well as from endogenous telomeric retrotransposons and TAS, in contrast to nontelomeric piRNA clusters. Of note, HP1 and H3K9me3 association with the EY00453 transgene (insTART) is not affected by $s p n E$ depletion, indicating that the chromatin status of this insertion region or particular TART copies is maintained by piRNA-independent mechanism.

It has been shown that maternal and/or zygotic piRNAs were sufficient to induce formation of repressive chromatin at non-telomeric piRNA clusters in early embryogenesis and that this state was maintained during germ cell development, even upon piRNA loss at later developmental stages [17]. In contrast, piRNAs are required at all stages of germline development to maintain telomere silencing. Accordingly, it was also reported that piRNA production from the dual-strand $42 A B$ piRNA cluster was far less sensitive to germline depletion of Rhi or HP1a compared to the subtelomeric piRNA clusters and transgene located in this region [34]. Thus, the chromatin dynamics of telomeric retrotransposons are more similar to those of the piRNA targets than of the "canonical" dual-strand piRNA clusters. At the same time, the telomeric regions bind Rhi and produce piRNA precursors from both genomic strands and are thus attributed to the dual-strand piRNA clusters.

We propose that the fundamental difference between Rhi-dependent telomeric and non-telomeric piRNA clusters is due to their different transcriptional regulation. Strong bidirectional promoters drive transcription of the telomeric retroelements $[39,57,58]$. The loss of piRNAs causes activation of the promoters in telomeres resulting 
in switching from a repressive to an active chromatin state $[25,58]$. In contrast, no discrete well-defined promoters were revealed within most of the heterochromatic non-telomeric piRNA clusters [59]. Transcription of certain clusters, such as $38 \mathrm{C} 1$, is initiated not only from internal initiation sites but also from flanking promoters, which differ significantly from canonical promoters [15]. Accordingly, the chromatin of all piRNA clusters we tested, including $38 \mathrm{C1}$, is resistant to piRNA loss. The TATA box-binding protein (TBP)-related factor 2 (TRF2) and Moonshiner, a paralog of a transcription factor IIA (TFIIA), are required for non-canonical transcription initiation and piRNA production from most of the nontelomeric clusters [59]. In contrast, we described TRF2 as a strong repressor of $H e T-A / T A H R E$ transcription, which is dispensable for $H e T-A$ small RNA production [60]. In fission yeast, high levels of transcriptional activity at the siRNA target locus prevent heterochromatin assembly apparently through the displacement of the silencing complex [61], thereby indicating that the transcription status of the locus and nature of promoters are important factors that influence chromatin remodeling caused by small RNAs.

We found that the clustered $H e T-A$ copies, normally positioned at the nuclear periphery, were located more toward the nuclear center following the loss of piRNAs. We suggest that this process is induced by the massive $H e T-A$ overexpression and is related to the expressiondependent nuclear positioning phenomenon described by several groups (for review see [62]). The state of telomeres resulting from piRNA loss can be defined as telomere dysfunction. It is believed that various signaling mechanisms from dysfunctional telomeres can link telomere integrity and cell cycle regulation [63]. We suggest that telomere dysfunction caused by piRNA loss is directly linked to the developmental defects observed in piRNA pathway mutants.

Telomere clustering at the nuclear envelope is a commonly observed but not absolute phenomenon. Telomere clustering in close proximity to the nuclear periphery was observed in the Drosophila somatic cells [64, 65]. However, telomeres are not clustered but do associate with the nuclear envelope in Drosophila oocytes at the pachytene stage of meiosis [66]. We revealed clustering of the $\mathrm{HeT}$ $A$ DNA FISH signals next to the nuclear periphery in the nuclei of polyploid nurse cells; however, it is unclear which particular telomeres are involved in clustering. piRNA loss affects the peripheral localization of telomeres in the germline but does not alter telomere clustering or the assembly of the telomere capping complex.

In addition to the telomeric regions, some recently transposed transcriptionally active TE copies inserted into euchromatin produce piRNAs [18]. Strong reduction in the H3K9me3 and Rhi association upon piwi depletion is observed for such TE copies [15]. The main difference between the telomeric arrays and individual TE copies is that the latter are targeted by piRNAs, mainly produced by other piRNA clusters or TE copies. We conclude that the telomeric piRNA clusters constitute a specific type of Rhi-dependent actively transcribed piRNA clusters, which are highly sensitive to the presence of piRNAs (Fig. 6a).

\section{Germline-specific chromatin structure of Drosophila telomeres}

Comparing the expression and chromatin structure of the telomeric transgenes in ovaries and somatic tissues reveals fundamental differences (Fig. 6b). Based on their ability to silence transgenes in somatic tissues, the TAS regions were defined as a heterochromatic domain, while the telomeric retrotransposon arrays were considered to be a transcriptionally active subdomain [30,31]. Remarkably, the subtelomeric regions of diverse organisms consist of highly variable sequences that exert a silencing effect on transgenes integrated within these regions [67]. Thus, the conserved silencing capacity of TAS is presumably important for telomere function. We observed the similar chromatin properties of TAS and terminal HeT-A-TART-TAHRE arrays in the Drosophila germline. Both telomeric regions produce piRNAs, bind Rhi, and are expressed at a similar low level (Table 1). Our data raise an intriguing question about the competition, or developmentally regulated replacement, of different chromatin complexes at TASs. PcG protein binding sites were revealed in TAS repeats [32]. Indeed, immunostaining and genetic analysis of the PcG protein mutants clearly demonstrate that the TAS zone serves as a platform for PcG protein-mediated chromatin assembly in somatic tissues [31, 32]. We suggest that initiation of the piRNA precursor transcription in TAS displaces the PcG complexes or prevents their deposition in the germline. Our data agree with the previous observation that Piwi negatively regulates PcG protein binding to chromatin and H3K27me3 levels in Drosophila ovaries [68]. Tissue-specific silencing mechanisms have been observed by other groups; for example, the Polycomb repressive complexes were shown to silence transgenes carrying the retrotransposon Idefix in somatic tissues but not in ovarian follicular cells [69]. Interestingly, the retrotransposon $m d g 1$ copies marked by H3K27me3 in the ovarian somatic cells were not susceptible to the piRNA-mediated transcriptional silencing [42]. Our results and previously published observations indicate that complex and competitive relationships between the various chromatin complexes define the chromatin structure of the genomic 
a

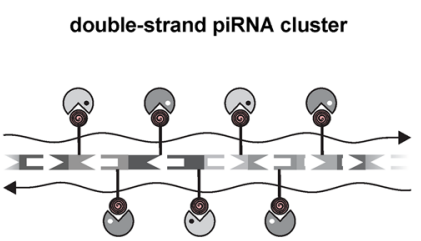

WILD-TYPE OVARIES

telomeric piRNA cluster

\section{PIRNA PATHWAY MUTANT OVARIES}
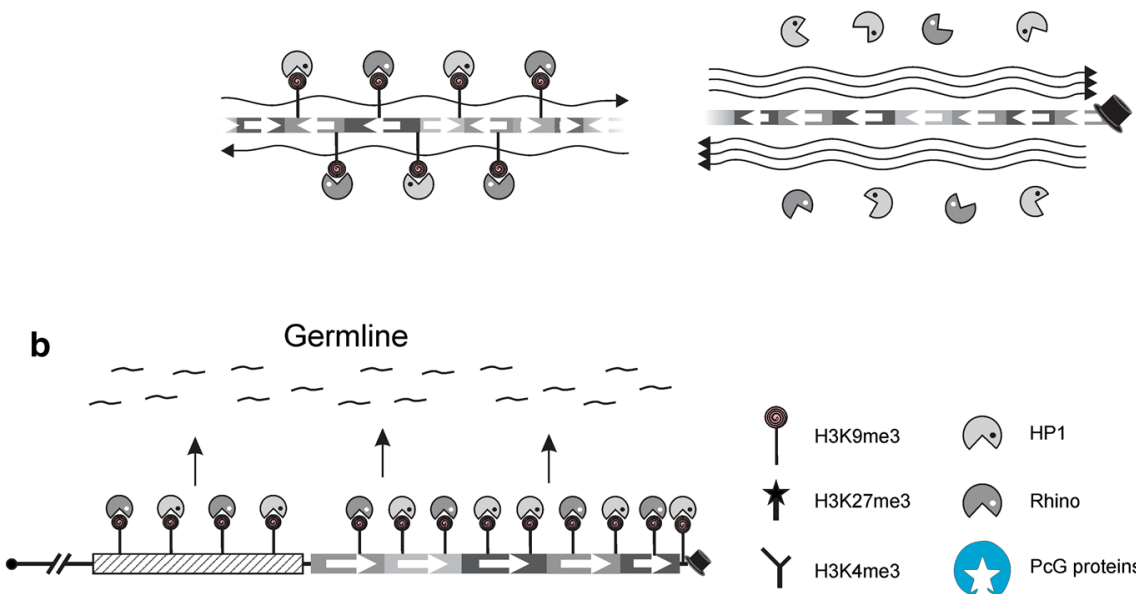

ค HP1

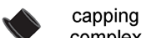

(2) Rhino

$\approx$ piRNAs

(1) PCG proteins

- centromere

Somatic tissues

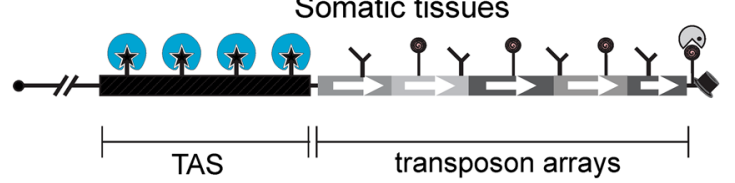

Fig. 6 Telomeres represent a distinct type of self-targeting dual-strand piRNA cluster. a Schematic representation of three types of dual-strand piRNA clusters. The chromatin structure of "canonical" dual-strand piRNA clusters is established by maternally inherited piRNAs but maintained by a piRNA-independent mechanism. On the contrary, piRNAs are strongly required for maintenance of the chromatin state of telomeric and euchromatic TE-associated piRNA clusters during oogenesis. Assembly of telomere protection capping complex is not affected by piRNAs. b Comparison of telomeric chromatin in somatic and germ cells. A schematic distribution of chromatin components along telomeric retrotransposon arrays and TAS is based on our study and previously published results [30, 31, 33]. In somatic tissues, TAS and HeT-A-TART-TAHRE arrays are subdivided into repressed and transcriptionally active domains, respectively. In the germline, both telomeric regions form piRNA cluster(s) enriched in HP1, H3K9me3, and Rhi

loci, including telomeres, particularly in the developmental context.

\section{Conclusions}

Our results demonstrate that the piRNA pathway is a robust mechanism of telomere homeostasis in the germline. piRNAs play a pivotal role in the establishment and maintenance of telomeric chromatin in the germline, facilitating loading of $\mathrm{HP} 1$ and $\mathrm{H} 3 \mathrm{~K} 9 \mathrm{me} 3$ at different telomeric regions. piRNA pathway disruption results in telomere dysfunction characterized by the loss of heterochromatic markers, overexpression of telomeric repeats, and translocation of telomeres from the periphery to the nuclear interior. In contrast to somatic tissues, where
TAS is a repressed domain, the HeT-A-TART-TAHRE arrays and TAS show similar chromatin structure and transcriptional status in the germline and belong to the Rhi-enriched piRNA-producing domain. However, strong heterogeneity in piRNA production and Rhi binding is observed along the telomeric transposon arrays. It is likely that TART retrotransposons are less susceptible to Rhi binding than $H e T-A$. The telomeric piRNA clusters belong to a specific type of Rhi-dependent piRNA clusters because telomeric retrotransposon transcripts driven by bidirectional promoters serve simultaneously as precursors of piRNAs and as their only targets. It is important that these transcripts are also used for telomere elongation in the germline. 


\section{Methods}

\section{Drosophila transgenic strains}

Transgenic strains EY08176, EY00453, EY00802, EY09966, and EY03383 carrying the EPgy2 element and inserted within different telomeric regions were described previously [30] and were kindly provided by J. Mason. Misy natural strain was obtained from the collection of Institut de Genetique Humaine (CNRS), Montpellier, France. P\{EPgy2\}Upf3 ${ }^{\text {EY03241 }}$ (stock \#16558) was obtained from the Bloomington Drosophila Stock Centre. Strains bearing spindle-E (spn-E) mutations were $r u^{1} s t^{1} s p n-E^{1} e^{1} c a^{1} / T M 3, S b^{1} e^{s}$ and $r u^{1} s t^{1} s p n-E^{h l s 3987}$ $e^{1} c a^{1} / T M 3, S b^{1} e^{s}$. We used piwi $i^{2}$ and $p i w i^{N t}$ alleles [70]. Zuc mutants were $z u c^{H m 27} / D f(2 L) P R L$ trans-heterozygous flies [49]. GLKD (from "germline knockdown") flies were F1 of the cross of two strains bearing constructs with short hairpin (sh) RNA (spnE_sh, \#103913, VDRC; piwi_sh, \#101658, VDRC) and strain \#25751 (P\{UASDcr-2.D $\} 1, \quad w^{1118}, \quad P\{G A L 4$-nos.NGT\}40, Bloomington Stock Center) providing GAL4 expression under the control of the germline-specific promoter of the nanos (nos) gene.

Fluorescence in situ hybridization (FISH) with polytene chromosomes was performed as previously described [71]. A PCR fragment amplified using white-specific primers $5^{\prime}$-catgatcaagacatctaaaggc- $3^{\prime}$ and $5^{\prime}$-gcaccgagcccgagttcaag- $3^{\prime}$ was labeled with a DIG DNA labeling kit (Roche).

\section{RT-PCR analysis}

RNA was isolated from the ovaries of 3-day-old females. cDNA was synthesized using random hexamers and SuperScript II reverse transcriptase (Life Technologies). cDNA samples were analyzed by real-time quantitative PCR using SYTO-13 dye on a Light Cycler 96 (Roche). Values were averaged and normalized to the expression level of the ribosomal protein gene rp49. Standard error of mean (SEM) for two independent RNA samples was calculated. The primers used are listed in Additional file 2: Table S4.

\section{Small RNA library preparation and analysis}

Small RNAs 19-29-nt in size from total ovarian RNA extracts were cloned as previously described [38]. Libraries were barcoded according to Illumina TrueSeq Small RNA sample prep kit instructions and submitted for sequencing using the Illumina HiSeq-2000 sequencing system. After clipping the Illumina 3 -adapter sequence, small RNA reads that passed quality control and minimal length filter (>18 nt) were mapped (allowing 0 mismatches) to the Drosophila melanogaster genome (Apr. 2006, BDGP assembly R5/dm3) or transgenes by bowtie [72]. Small RNA libraries were normalized to 1 Mio sequenced reads. The plotting of size distributions, read coverage, and nucleotide biases were performed as described previously [20]. Ovarian small RNA-seq data for $y^{1} w^{67 c 23}$ and transgenic strains EY08176, EY00453, EY00802, EY09966, EY03383, and EY03241 were deposited at the Gene Expression Omnibus (GEO), accession number GSE98981.

\section{Chromatin immunoprecipitation}

For every IP experiment $\sim 200$ pairs of ovaries were dissected. ChIP was performed according to the published procedure [73]. Chromatin was immunoprecipitated with the following antibodies: anti-HP1a (C1A9, Developmental Studies Hybridoma Bank), anti-trimethyl-histone H3 Lys9 (Millipore), Rhi antiserum [58]. Primers used in the study are listed in Additional file 2: Table S4. Quantitative PCR was conducted with a Light cycler 96 (Roche). Standard error of mean (SEM) of triplicate PCR measurements for three-six biological replicates was calculated.

\section{FISH and immunostaining}

The combined evaluation of protein and DNA localization was done according to the previously described procedure [71]. Rabbit anti-H3K27me3 (Abcam) and rat anti-Rhi antibodies [58] were used. The probes used for DNA FISH were: TART, cloned fragment of TART-A ORF2 corresponding to 434-2683 nucleotides in GenBank sequence DMU02279; $H e T-A$, cloned fragment of $H e T-A$ ORF corresponding to 1746-4421 nucleotides in GenBank sequence DMU 06920; TAHRE, PCR fragment corresponding to 5147-6165 nucleotides in GenBank sequence AJ542581. The TART probe was labeled using a DIG DNA labeling kit (Roche), $H e T-A$ by a Bio-Nick labeling system (Invitrogen), and TAHRE by PCR DIG labeling mix (Roche). Probes corresponding to $2 \mathrm{R}-3 \mathrm{R}$ TAS, 2L-3L TAS, and $42 A B$ regions were PCR fragments obtained with primers listed in Additional file 2: Table S4 and labeled with a PCR DIG DNA labeling mix (Roche). To stain DNA, ovaries were incubated in PBS containing $0.5 \mu \mathrm{g} / \mathrm{ml}$ DAPI. Three biological replicates were obtained for each experiment. Zeiss LSM 510 Meta and Olympus FV10i confocal microscopes were used for visualization. Confocal image z-stacks were generated with a slice step of $1.05 \mu \mathrm{M}$.

\section{Calculations of distance from the clustered HeT-A DNA FISH spots to the nuclear periphery}

Calculations were performed using Imaris 7.4.2 software with manual segmentation of nuclei based on DAPI staining, automatic segmentation of in situ signal spots, and automatic calculation of a center of homogeneous 
mass corresponding to the main $H e T-A$ cluster. FISH spot size is the diameter of a sphere encompassing all of the spots in the $\mathrm{XY}$ plane and $\mathrm{Z}$ position corresponding to the center of mass. The distance between the center of image masses and the nearest point on the nuclear surface was measured by increasing the radius of a sphere originated from the center of image masses until it intersected with the nuclear surface and later recording the radius as a distance. An independent two-sample $t$ test was used to compare hetero- and trans-heterozygous mutants.

\section{Northern blot of small RNAs}

Northern analysis of small RNAs was performed as previously described [20]. The white sense probe contained a cloned PCR fragment amplified using primers $5^{\prime}$-ctcacctatgcctggcacaatatg- $3^{\prime}$ and $5^{\prime}$-attcagcagggtcgtctttccg- $3^{\prime}$. Hybridization with $\mathrm{P}^{32} 5^{\prime}$-end-labeled oligonucleotide $5^{\prime}$-actcgtcaaaatggctgtgata- $3^{\prime}$ complementary to the miRNA-13b-1 was used as a loading control. The blots were visualized with a phosphorimager Typhoon FLA9500 (Amersham). Northern blot quantification was done using ImageJ.

\section{Additional files}

Additional file 1: Figure S1. Localization of telomeric transgenes. Figure S2. Profiles of telomeric retroelement small RNAs (related to Fig. 1a). Figure S3. Generation of small RNAs by telomeric transgenes (related to Fig. 1c). Figure S4. Quantification of Northern blots of small RNAs in transgenic strains (related to Fig. 1f). Figure S5. Rhi and HP1 occupancy at telomeric transgenes (related to Fig. 2). Figure S6. Expression of EY08176 telomeric transgene is increased in ovaries of the spnE mutants. Figure S7. Nuclear localization of telomeres. Figure S8. Subtelomeric chromatin in the germline (related to Fig. 5b).

Additional file 2: Table S1. Small RNA mapping to the telomeric and euchromatic transgenes. Table S2. Colocalization of HeT-A, TART and TAS with Rhino. Table S3. Colocalization of TAS with H3K27me3 in the Drosophila germline. Table S4. Primers used in the study (5'-to-3').

\section{Authors' contributions}

ER performed ChIP and RT-PCR, VM and SIG performed FISH and immunostaining, NA and PAK performed small RNA libraries preparation, NA did northern blot analysis, SR performed small RNA-seq data analysis, YA performed fly crossings and fly stocks maintenance, SL performed distance calculations from the HeT-A DNA FISH spots to the nuclear periphery, ER, IO participated in manuscript editing and result visualization, AK planned experiments, contributed to the data analysis, and wrote the paper. All authors read and approved the final manuscript.

\section{Author details}

${ }^{1}$ Institute of Molecular Genetics, Russian Academy of Sciences, Kurchatov sq 2, Moscow, Russia 123182. ${ }^{2}$ Department of Biochemistry, Faculty of Biology, Lomonosov Moscow State University, Moscow, Russia 119991.

\section{Acknowledgements}

The authors are grateful to J. Mason for transgenic D. melanogaster strains, Y. Schwartz for critical comments on the manuscript, Y. Rong for HOAP antibodies, and O. Olenkina for consultations on genetic crosses. We thank Dr. M. Semenova and User Facilities Centre of M.V. Lomonosov Moscow State
University as well as User Centre of the Institute of Molecular Genetics, RAS, for the provided opportunity to use microscopy equipment.

\section{Competing interests}

The authors declare that they have no competing interests.

\section{Availability of data and materials}

Ovarian small RNA-seq data for $y^{1} w^{67 c 23}$ and transgenic strains EY08176, EY00453, EY00802, EY09966, ET03383 and EY03241 were deposited to the Gene Expression Omnibus (GEO), Accession Number GSE98981.

\section{Consent for publication}

Not applicable.

Ethics approval and consent to participate

Not applicable.

\section{Funding}

This work was supported in part by the Russian Science Foundation (Grant No. 16-14-10167), the Russian Foundation for Basic Researches (Grant No. 16-0401107 to S.G.), and the Skoltech Systems Biology Fellowship to S.R.

\section{Publisher's Note}

Springer Nature remains neutral with regard to jurisdictional claims in published maps and institutional affiliations.

Received: 24 April 2018 Accepted: 6 July 2018

Published online: 12 July 2018

\section{References}

1. Azzalin CM, Lingner J. Telomere functions grounding on TERRA firma. Trends Cell Biol. 2015;25(1):29-36.

2. Cao F, Li X, Hiew S, Brady H, Liu Y, Dou Y. Dicer independent small RNAs associate with telomeric heterochromatin. RNA. 2009;15(7):1274-81.

3. Savitsky M, Kwon D, Georgiev P, Kalmykova A, Gvozdev V. Telomere elongation is under the control of the RNAi-based mechanism in the Drosophila germline. Genes Dev. 2006;20(3):345-54.

4. Tatsuke T, Sakashita K, Masaki Y, Lee JM, Kawaguchi Y, Kusakabe T. The telomere-specific non-LTR retrotransposons SART1 and TRAS1 are suppressed by Piwi subfamily proteins in the silkworm, Bombyx mori. Cell Mol Biol Lett. 2010;15(1):118-33.

5. Vrbsky J, Akimcheva S, Watson JM, Turner TL, Daxinger L, Vyskot B, et al. siRNA-mediated methylation of Arabidopsis telomeres. PLoS Genet. 2010;6(6):e1000986.

6. Sugiyama T, Cam HP, Sugiyama R, Noma K, Zofall M, Kobayashi R, et al. SHREC, an effector complex for heterochromatic transcriptional silencing. Cell. 2007;128(3):491-504

7. Iwasaki YW, Siomi MC, Siomi H. PIWI-Interacting RNA: its Biogenesis and Functions. Annu Rev Biochem. 2015;84:405-33.

8. van Wolfswinkel JC. Piwi and potency: PIWI proteins in animal stem cells and regeneration. Integr Comp Biol. 2014;54(4):700-13.

9. Toth KF, Pezic D, Stuwe E, Webster A. The piRNA pathway guards the germline genome against transposable elements. Adv Exp Med Biol. 2016;886:51-77.

10. Brennecke J, Aravin AA, Stark A, Dus M, Kellis M, Sachidanandam R, et al. Discrete small RNA-generating loci as master regulators of transposon activity in Drosophila. Cell. 2007;128(6):1089-103.

11. Chen YC, Stuwe E, Luo Y, Ninova M, Le Thomas A, Rozhavskaya E, et al. Cutoff suppresses RNA Polymerase II termination to ensure expression of piRNA precursors. Mol Cell. 2016;63(1):97-109.

12. Hur JK, Luo Y, Moon S, Ninova M, Marinov GK, Chung YD, et al. Splicingindependent loading of TREX on nascent RNA is required for efficient expression of dual-strand piRNA clusters in Drosophila. Genes Dev. 2016;30(7):840-55

13. Zhang F, Wang J, Xu J, Zhang Z, Koppetsch BS, Schultz N, et al. UAP56 couples piRNA clusters to the perinuclear transposon silencing machinery. Cell. 2012;151(4):871-84. 
14. Klattenhoff C, Xi H, Li C, Lee S, Xu J, Khurana JS, et al. The Drosophila HP1 homolog Rhino is required for transposon silencing and piRNA production by dual-strand clusters. Cell. 2009;138(6):1137-49.

15. Mohn F, Sienski G, Handler D, Brennecke J. The rhino-deadlock-cutoff complex licenses noncanonical transcription of dual-strand piRNA clusters in Drosophila. Cell. 2014;157(6):1364-79.

16. Zhang Z, Wang J, Schultz N, Zhang F, Parhad SS, Tu S, et al. The HP1 homolog rhino anchors a nuclear complex that suppresses piRNA precursor splicing. Cell. 2014;157(6):1353-63.

17. Akkouche A, Mugat B, Barckmann B, Varela-Chavez C, Li B, Raffel R, et al. Piwi is required during Drosophila embryogenesis to license dual-strand piRNA clusters for transposon repression in adult ovaries. Mol Cell. 2017:66:411.

18. Shpiz S, Ryazansky S, Olovnikov I, Abramov Y, Kalmykova A. Euchromatic transposon insertions trigger production of novel $\mathrm{Pi}$ - and endo-siRNAs at the target sites in the Drosophila germline. PLoS Genet. 2014;10(2):e1004138.

19. de Vanssay A, Bouge AL, Boivin A, Hermant C, Teysset L, Delmarre V, et al. Paramutation in Drosophila linked to emergence of a piRNA-producing locus. Nature. 2012:490(7418):112-5.

20. Olovnikov I, Ryazansky S, Shpiz S, Lavrov S, Abramov Y, Vaury C, et al. De novo piRNA cluster formation in the Drosophila germ line triggered by transgenes containing a transcribed transposon fragment. Nucleic Acids Res. 2013:41(11):5757-68.

21. Garavis M, Gonzalez C, Villasante A. On the origin of the eukaryotic chromosome: the role of noncanonical DNA structures in telomere evolution. Genome Biol Evol. 2013:5(6):1142-50.

22. Abad JP, De Pablos B, Osoegawa K, De Jong PJ, Martin-Gallardo A, Villasante A. Genomic analysis of Drosophila melanogaster telomeres: full-length copies of HeT-A and TART elements at telomeres. Mol Biol Evol. 2004:21(9):1613-9.

23. Pardue ML, DeBaryshe PG. Retrotransposons provide an evolutionarily robust non-telomerase mechanism to maintain telomeres. Annu Rev Genet. 2003;37:485-511.

24. Abad JP, De Pablos B, Osoegawa K, De Jong PJ, Martin-Gallardo A, Villasante A. TAHRE, a novel telomeric retrotransposon from Drosophila melanogaster, reveals the origin of Drosophila telomeres. Mol Biol Evol. 2004:21(9):1620-4.

25. Shpiz S, Olovnikov I, Sergeeva A, Lavrov S, Abramov Y, Savitsky M, et al. Mechanism of the piRNA-mediated silencing of Drosophila telomeric retrotransposons. Nucleic Acids Res. 2011;39(20):8703-11.

26. Ryazansky S, Radion E, Mironova A, Akulenko N, Abramov Y, Morgunova V, et al. Natural variation of piRNA expression affects immunity to transposable elements. PLoS Genet. 2017;13(4):e1006731.

27. Czech B, Preall JB, McGinn J, Hannon GJ. A transcriptome-wide RNAi screen in the Drosophila ovary reveals factors of the germline piRNA pathway. Mol Cell. 2013;50(5):749-61.

28. Klenov MS, Lavrov SA, Stolyarenko AD, Ryazansky SS, Aravin AA, Tuschl T, et al. Repeat-associated siRNAs cause chromatin silencing of retrotransposons in the Drosophila melanogaster germline. Nucleic Acids Res 2007:35(16):5430-8

29. Rozhkov NV, Hammell M, Hannon GJ. Multiple roles for Piwi in silencing Drosophila transposons. Genes Dev. 2013;27(4):400-12.

30. Biessmann H, Prasad S, Semeshin VF, Andreyeva EN, Nguyen Q, Walter MF, et al. Two distinct domains in Drosophila melanogaster telomeres. Genetics. 2005;171(4):1767-77.

31. Andreyeva EN, Belyaeva ES, Semeshin VF, Pokholkova GV, Zhimulev IF. Three distinct chromatin domains in telomere ends of polytene chromosomes in Drosophila melanogaster Tel mutants. J Cell Sci. 2005;1 18(Pt 23):5465-77.

32. Boivin A, Gally C, Netter S, Anxolabehere D, Ronsseray S. Telomeric associated sequences of Drosophila recruit polycomb-group proteins in vivo and can induce pairing-sensitive repression. Genetics. 2003;164(1):195-208.

33. Silva-Sousa R, Casacuberta E. The JIL-1 kinase affects telomere expression in the different telomere domains of Drosophila. PLOS ONE. 2013;8(11):e81543.

34. Marie PP, Ronsseray S, Boivin A. From embryo to adult: piRNA-Mediated silencing throughout germline development in Drosophila. G3 (Bethesda). 2017;7(2):505-16.
35. Josse T, Teysset L, Todeschini AL, Sidor CM, Anxolabehere D, Ronsseray S. Telomeric trans-silencing: an epigenetic repression combining RNA silencing and heterochromatin formation. PLoS Genet. 2007;3(9):1633-43.

36. Todeschini AL, Teysset L, Delmarre V, Ronsseray S. The epigenetic transsilencing effect in Drosophila involves maternally-transmitted small RNAs whose production depends on the piRNA pathway and HP1. PLoS ONE. 2010;5(6):e11032.

37. Le Thomas A, Stuwe E, Li S, Du J, Marinov G, Rozhkov N, et al. Transgenerationally inherited piRNAs trigger piRNA biogenesis by changing the chromatin of piRNA clusters and inducing precursor processing. Genes Dev. 2014;28(15):1667-80.

38. Muerdter F, Olovnikov I, Molaro A, Rozhkov NV, Czech B, Gordon A, et al. Production of artificial piRNAs in flies and mice. RNA. 2012;18(1):42-52.

39. Maxwell PH, Belote JM, Levis RW. Identification of multiple transcription initiation, polyadenylation, and splice sites in the Drosophila melanogaster TART family of telomeric retrotransposons. Nucleic Acids Res. 2006;34(19):5498-507.

40. Gunawardane LS, Saito K, Nishida KM, Miyoshi K, Kawamura Y, Nagami T, et al. A slicer-mediated mechanism for repeat-associated siRNA $5^{\prime}$ end formation in Drosophila. Science. 2007:315(5818):1587-90.

41. Le Thomas A, Rogers AK, Webster A, Marinov GK, Liao SE, Perkins EM, et al. Piwi induces piRNA-guided transcriptional silencing and establishment of a repressive chromatin state. Genes Dev. 2013;27(4):390-9.

42. Sienski G, Donertas D, Brennecke J. Transcriptional silencing of transposons by piwi and maelstrom and its impact on chromatin state and gene expression. Cell. 2012;151(5):964-80.

43. Wang SH, Elgin SC. Drosophila Piwi functions downstream of piRNA production mediating a chromatin-based transposon silencing mechanism in female germ line. Proc Natl Acad Sci USA. 2011;108(52):21164-9.

44. Volpe AM, Horowitz H, Grafer CM, Jackson SM, Berg CA. Drosophila rhino encodes a female-specific chromo-domain protein that affects chromosome structure and egg polarity. Genetics. 2001;159(3):1117-34.

45. Garcia-Cao M, O'Sullivan R, Peters AH, Jenuwein T, Blasco MA. Epigenetic regulation of telomere length in mammalian cells by the Suv39h1 and Suv39h2 histone methyltransferases. Nat Genet. 2004;36(1):94-9.

46. Fanti L, Dorer DR, Berloco M, Henikoff S, Pimpinelli S. Heterochromatin protein 1 binds transgene arrays. Chromosoma. 1998;107(5):286-92.

47. Perrini B, Piacentini L, Fanti L, Altieri F, Chichiarelli S, Berloco M, et al. HP1 controls telomere capping, telomere elongation, and telomere silencing by two different mechanisms in Drosophila. Mol Cell. 2004;15(3):467-76.

48. Malone CD, Brennecke J, Dus M, Stark A, McCombie WR, Sachidanandam $R$, et al. Specialized piRNA pathways act in germline and somatic tissues of the Drosophila ovary. Cell. 2009;137(3):522-35.

49. Pane A, Wehr K, Schupbach T. zucchini and squash encode two putative nucleases required for rasiRNA production in the Drosophila germline. Dev Cell. 2007;12(6):851-62

50. Cenci G, Siriaco G, Raffa GD, Kellum R, Gatti M. The Drosophila HOAP protein is required for telomere capping. Nat Cell Biol. 2003;5(1):82-4.

51. Khurana JS, Xu J, Weng Z, Theurkauf WE. Distinct functions for the Drosophila piRNA pathway in genome maintenance and telomere protection. PLoS Genet. 2010;6(12):e1001246.

52. Karpen GH, Spradling AC. Analysis of subtelomeric heterochromatin in the Drosophila minichromosome Dp1187 by single P element insertional mutagenesis. Genetics. 1992;132(3):737-53.

53. Wallrath LL, Elgin SC. Position effect variegation in Drosophila is associated with an altered chromatin structure. Genes Dev. 1995;9(10):1263-77.

54. Danilevskaya ON, Traverse KL, Hogan NC, DeBaryshe PG, Pardue ML. The two Drosophila telomeric transposable elements have very different patterns of transcription. Mol Cell Biol. 1999;19(1):873-81.

55. George JA, DeBaryshe PG, Traverse KL, Celniker SE, Pardue ML. Genomic organization of the Drosophila telomere retrotransposable elements. Genome Res. 2006;16(10):1231-40.

56. Savitsky M, Kravchuk O, Melnikova L, Georgiev P. Heterochromatin protein 1 is involved in control of telomere elongation in Drosophila melanogaster. Mol Cell Biol. 2002;22(9):3204-18.

57. Danilevskaya ON, Arkhipova IR, Traverse KL, Pardue ML. Promoting in tandem: the promoter for telomere transposon HeT-A and implications for the evolution of retroviral LTRs. Cell. 1997:88(5):647-55.

58. Radion E, Ryazansky S, Akulenko N, Rozovsky Y, Kwon D, Morgunova V et al. Telomeric retrotransposon HeT-A contains a bidirectional promoter 
that initiates divergent transcription of piRNA precursors in Drosophila germline. J Mol Biol. 2017;429(21):3280-9.

59. Andersen PR, Tirian L, Vunjak M, Brennecke J. A heterochromatindependent transcription machinery drives piRNA expression. Nature. 2017;549(7670):54-9.

60. Morgunova V, Akulenko N, Radion E, Olovnikov I, Abramov Y, Olenina LV et al. Telomeric repeat silencing in germ cells is essential for early development in Drosophila. Nucleic Acids Res. 2015;43(18):8762-73.

61. Shimada Y, Mohn F, Buhler M. The RNA-induced transcriptional silencing complex targets chromatin exclusively via interacting with nascent transcripts. Genes Dev. 2016;30(23):2571-80.

62. Shevelyov YY, Nurminsky DI. The nuclear lamina as a gene-silencing hub. Curr Issues Mol Biol. 2012;14(1):27-38.

63. Ye J, Renault VM, Jamet K, Gilson E. Transcriptional outcome of telomere signalling. Nat Rev Genet. 2014;15(7):491-503.

64. Wesolowska N, Amariei FL, Rong YS. Clustering and protein dynamics of Drosophila melanogaster telomeres. Genetics. 2013;195(2):381-91.

65. Marshall WF, Dernburg AF, Harmon B, Agard DA, Sedat JW. Specific interactions of chromatin with the nuclear envelope: positional determination within the nucleus in Drosophila melanogaster. Mol Biol Cell. 1996;7(5):825-42.

66. Lake CM, Hawley RS. The molecular control of meiotic chromosomal behavior: events in early meiotic prophase in Drosophila oocytes. Annu Rev Physiol. 2012;74:425-51.
67. Mefford HC, Trask BJ. The complex structure and dynamic evolution of human subtelomeres. Nat Rev Genet. 2002;3(2):91-102.

68. Peng JC, Valouev A, Liu N, Lin H. Piwi maintains germline stem cells and oogenesis in Drosophila through negative regulation of Polycomb group proteins. Nat Genet. 2016;48(3):283-91.

69. Dufourt J, Brasset E, Desset S, Pouchin P, Vaury C. Polycomb groupdependent, heterochromatin protein 1-independent, chromatin structures silence retrotransposons in somatic tissues outside ovaries. DNA Res. 2011:18(6):451-61.

70. Klenov MS, Sokolova OA, Yakushev EY, Stolyarenko AD, Mikhaleva EA, Lavrov SA, et al. Separation of stem cell maintenance and transposon silencing functions of Piwi protein. Proc Natl Acad Sci USA. 2011;108(46):18760-5.

71. Lavrov S, Dejardin J, Cavalli G. Combined immunostaining and FISH analysis of polytene chromosomes. Methods Mol Biol. 2004;247:289-303.

72. Langmead B, Trapnell C, Pop M, Salzberg SL. Ultrafast and memoryefficient alignment of short DNA sequences to the human genome. Genome Biol. 2009:10(3):R25.

73. Akulenko N, Ryazansky S, Morgunova V, Komarov PA, Olovnikov I, Vaury $C$, et al. Transcriptional and chromatin changes accompanying de novo formation of transgenic piRNA clusters. RNA. 2018;24:574-84.
Ready to submit your research? Choose BMC and benefit from:

- fast, convenient online submission

- thorough peer review by experienced researchers in your field

- rapid publication on acceptance

- support for research data, including large and complex data types

- gold Open Access which fosters wider collaboration and increased citations

- maximum visibility for your research: over 100M website views per year

At BMC, research is always in progress.

Learn more biomedcentral.com/submissions 